DFF $1 / 9 / 02$

\title{
Solitons for the fuzzy sphere from matrix model
}

\author{
P. Valtancoli \\ Dipartimento di Fisica, Polo Scientifico Universitá di Firenze \\ and INFN, Sezione di Firenze (Italy) \\ Via G. Sansone 1, 50019 Sesto Fiorentino, Italy
}

\begin{abstract}
Recently we have introduced a matrix model depending on two coupling constants $g^{2}$ and $\lambda$, which contains the fuzzy sphere as a background; to obtain the classical limit $g^{2}$ must depend on $N$ in a precise way. In this paper we show how to obtain the classical solitons of the $N \rightarrow \infty$ limit imposing the development $\lambda=\frac{1}{2}+\frac{\lambda_{0}}{N}$; as a consequence at finite $N$ one obtains a noncommutative version of the solitons for the fuzzy sphere.
\end{abstract}




\section{Introduction}

Recently, noncommutative gauge theories [1]-[2]-[3] on a noncommutative sphere have been studied by expanding a matrix model [4]-[5]-[6]-[7]-[8]-[9] around its classical solution [10][11]- [12]-[13]- [14]. The fuzzy sphere is considered as a classical background, and the fluctuations on the background are the fields of noncommutative gauge theory. In a previous paper [14] we have introduced a matrix model which depends on two coupling constants, $g^{2}$ and $\lambda$ :

$$
S(\lambda)=S_{0}+\lambda S_{1}=-\frac{1}{g^{2}} \operatorname{Tr}\left[\frac{1}{4}\left[A_{i}, A_{j}\right]\left[A^{i}, A^{j}\right]-\frac{2}{3} i \lambda \rho \epsilon^{i j k} A_{i} A_{j} A_{k}+\rho^{2}(1-\lambda) A^{i} A_{i}\right] .
$$

We looked for other classical solutions besides the fuzzy sphere and found them for $\lambda<1$. These make the fuzzy sphere solution unstable for $\lambda<\frac{1}{2}$ and stable otherwise.

In this paper we answer a question left in the previous one, i.e. whether this new class of classical solutions leads to solitons for the noncommutative gauge theory on the fuzzy sphere.

Generally a solution of the matrix model could be defined as a soliton, but we have to be more restrictive, i.e. to call a noncommutative soliton a configuration which tends to a classical soliton of the sphere in the $N \rightarrow \infty$ limit.

What is called fluctuation is the difference between the fuzzy sphere $\hat{x}^{i}$ and the new class of classical solutions $A^{i}$, but to recover a well-defined classical limit, the fluctuations must go to zero as $\frac{1}{N}$, in the $N \rightarrow \infty$ limit [10]-[12]. Such property distinguishes what can be called a field of noncommutative gauge theory from something indefinite. Therefore many of the classical solutions of the matrix model are meaningless, also if they can make the fuzzy sphere background unstable [14].

To find a good fluctuation, we need to explore the neighborhood of $\lambda=\frac{1}{2}$ ( precisely

$\lambda=\frac{1}{2}+\frac{\lambda_{0}}{N}$ ), since then our new class of classical solutions is a continuous deformation of the fuzzy sphere, and the difference between them is of order $\frac{1}{N}$.

It was already clear from [10]-[12] that the coupling constant $g^{2}$ must scale in a precise way with $N$ to recover a classical gauge theory on a sphere; the idea of the present article is to introduce a double scaling limit, for both $g^{2}$ and $\lambda$, to recover the classical solitons on the sphere from the classical solutions of the matrix model.

To verify that we have found the noncommutative solitons of the fuzzy sphere, we check that the gauge field and the scalar field defined from the fluctuations, obtained from the difference of two solutions of the matrix model, indeed satisfy the classical equations of motion, in the $N \rightarrow \infty$ limit. 
What we find is that, as typical for the case of solitons, an equation of motion for the gauge field is already present into the action as a quadratic term, while the other equation for the scalar field is equal to the eigenvalue problem for the $L^{2}$ operator. The only possible solutions of this equation are the spherical harmonics $Y_{l}^{m}(\Omega)$, and in our case it turns out that the scalar field is a combination of $Y_{l}^{m}(\Omega)$ with $l=2$. To find noncommutative solitons with higher spherical harmonics $l>2$, we conclude that one should explore models with $\lambda=\frac{3-l}{2}$. By analogy with the $\lambda=\frac{1}{2}$ case treated here, one should start from solutions of matrix model obtained with more general ansatze

$$
A_{i}+A_{i}^{j_{1}, \ldots, j_{l-1}} L_{j_{1}} \ldots L_{j_{l-1}}
$$

hoping that, around $\lambda=\frac{3-l}{2}$, these solutions are smooth deformations of the fuzzy sphere. To conclude our analysis, we evaluate the action of the matrix model at $\lambda=\frac{1}{2}+\frac{\lambda_{0}}{N}$, and compare it with the value of the action of the gauge theory on the sphere, at $\lambda=\frac{1}{2}$, and find complete agreement.

\section{Solutions of the matrix model for $\lambda=\frac{1}{2}+\frac{\lambda_{0}}{N}$}

In a recent paper [14] we have introduced an action depending on two coupling constants, $g^{2}$ and $\lambda$, which contains as a solution the fuzzy sphere

$$
S(\lambda)=S_{0}+\lambda S_{1}=-\frac{1}{g^{2}} \operatorname{Tr}\left[\frac{1}{4}\left[A_{i}, A_{j}\right]\left[A_{i}, A_{j}\right]-\frac{2}{3} i \rho \epsilon^{i j k} A_{i} A_{j} A_{k}+\rho^{2}(1-\lambda) A_{i} A_{i}\right] .
$$

The fuzzy sphere [15]-16]-17]-18]-19]-20] is a noncommutative manifold represented by the following algebra:

$$
\left[\hat{x}_{i}, \hat{x}_{j}\right]=i \rho \epsilon^{i j k} \hat{x}_{k} \quad \hat{x}^{i}=\rho L^{i}
$$

The radius of the sphere, obtained by the following condition,

$$
\hat{x}_{i} \hat{x}_{i}=R^{2}=\rho^{2} L^{i} L^{i}=\rho^{2} \frac{N(N+2)}{4}
$$

is kept fixed in the commutative limit $N \rightarrow \infty$, therefore

$$
\rho \sim \frac{1}{N}
$$


The equations of motion for the matrix model action $S(\lambda)$ contain other solutions rather than the fuzzy sphere, as found in [14]. Our aim is to use these solutions to construct solitons solutions for the fuzzy sphere [21]-[22]- 223]-[24]- 25]. Let us recall them, starting from the equations of motion

$$
\left[A^{j},\left[A^{i}, A^{j}\right]\right]-i \rho \lambda \epsilon^{i j k}\left[A^{j}, A^{k}\right]+2 \rho^{2}(1-\lambda) A^{i}=0 .
$$

Parameterizing the solution as

$$
A^{i}=A_{k}^{i} L^{k}
$$

the coefficients $A_{k}^{i}$ represent three arbitrary vectors $A_{i}^{1}, A_{i}^{2}, A_{i}^{3}$ that are constrained by the equations

$$
\begin{aligned}
& \left(2 \rho^{2}(1-\lambda)-\beta^{2}-\gamma^{2}\right) A_{i}^{1}+\left(A^{1} \cdot A^{2}\right) A_{i}^{2}+\left(A^{1} \cdot A^{3}\right) A_{i}^{3}+2 \lambda \rho \epsilon_{i j k} A_{j}^{2} A_{k}^{3}=0 \\
& \left(2 \rho^{2}(1-\lambda)-\alpha^{2}-\gamma^{2}\right) A_{i}^{2}+\left(A^{1} \cdot A^{2}\right) A_{i}^{1}+\left(A^{2} \cdot A^{3}\right) A_{i}^{3}+2 \lambda \rho \epsilon_{i j k} A_{j}^{3} A_{k}^{1}=0 \\
& \left(2 \rho^{2}(1-\lambda)-\alpha^{2}-\beta^{2}\right) A_{i}^{3}+\left(A^{1} \cdot A^{3}\right) A_{i}^{1}+\left(A^{2} \cdot A^{3}\right) A_{i}^{2}+2 \lambda \rho \epsilon_{i j k} A_{j}^{1} A_{k}^{2}=0
\end{aligned}
$$

where

$$
\left(A_{i}^{1}\right)^{2}=\alpha^{2} \quad\left(A_{i}^{2}\right)^{2}=\beta^{2} \quad\left(A_{i}^{3}\right)^{2}=\gamma^{2} .
$$

Without loss of generalities, we can choose the three vectors as follows:

$$
\begin{aligned}
& A_{x}^{1}=\alpha \quad A_{y}^{1}=A_{z}^{1}=0 \\
& A_{x}^{2}=\beta \cos \theta_{12} \quad A_{y}^{2}=\beta \sin \theta_{12} \quad A_{z}^{2}=0 \\
& A_{x}^{3}=\gamma \operatorname{cs} \theta_{13} \quad A_{y}^{3}=\gamma \sin \theta_{13} \sin \phi \quad A_{z}^{3}=\gamma \sin \theta_{13} \cos \phi .
\end{aligned}
$$

Apart from the fuzzy sphere, there is another class of solutions to the equations (2.7), characterized by the unique constraint:

$$
\alpha^{2}+\beta^{2}+\gamma^{2}=4 \rho^{2}(1-\lambda)+4 \lambda^{2} \rho^{2}
$$

and by the parameterizations 


$$
\begin{aligned}
& \cos \phi=-\frac{2 \lambda \alpha \rho}{\sqrt{\left(2 \rho^{2}(1-\lambda)-\beta^{2}\right)\left(2 \rho^{2}(1-\lambda)-\gamma^{2}\right)+4 \lambda^{2} \alpha^{2} \rho^{2}}} \\
& \sin \phi=\sqrt{\frac{\left(2 \rho^{2}(1-\lambda)-\beta^{2}\right)\left(2 \rho^{2}(1-\lambda)-\gamma^{2}\right)}{\left(2 \rho^{2}(1-\lambda)-\beta^{2}\right)\left(2 \rho^{2}(1-\lambda)-\gamma^{2}\right)+4 \lambda^{2} \alpha^{2} \rho^{2}}} \\
& \cos \theta_{12}=-\frac{1}{\alpha \beta} \sqrt{\left(2 \rho^{2}(1-\lambda)-\alpha^{2}\right)\left(2 \rho^{2}(1-\lambda)-\beta^{2}\right)} \\
& \sin \theta_{12}=\frac{1}{\alpha \beta} \sqrt{2 \rho^{2}(1-\lambda)\left(\alpha^{2}+\beta^{2}-2 \rho^{2}(1-\lambda)\right)} \\
& \cos \theta_{13}=-\frac{1}{\alpha \gamma} \sqrt{\left(2 \rho^{2}(1-\lambda)-\alpha^{2}\right)\left(2 \rho^{2}(1-\lambda)-\gamma^{2}\right)} \\
& \sin \theta_{13}=-\frac{1}{\alpha \gamma} \sqrt{2 \rho^{2}(1-\lambda)\left(\alpha^{2}+\gamma^{2}-2 \rho^{2}(1-\lambda)\right.} \\
& \cos \theta_{23}=-\frac{1}{\beta \gamma} \sqrt{\left.2 \rho^{2}(1-\lambda)-\beta^{2}\right)\left(2 \rho^{2}(1-\lambda)-\gamma^{2}\right)} \\
& \sin \theta_{23}=-\frac{1}{\beta \gamma} \sqrt{2 \rho^{2}(1-\lambda)\left(\beta^{2}+\gamma^{2}-2 \rho^{2}(1-\lambda)\right)}
\end{aligned}
$$

where $\cos \theta_{23}=\cos \theta_{12} \cos \theta_{13}+\sin \theta_{12} \sin \theta_{13} \sin \phi$.

The basic requirement to construct solitons solutions is developing the matrix $A^{i}$ as a background plus fluctuations :

$$
A_{i}=\hat{x}_{i}+\rho R \hat{a}_{i}
$$

In front of the $U(1)$ noncommutative connection $\hat{a}_{i}$ there is a factor $\rho$, which means that in the $N \rightarrow \infty$ limit the difference between $A_{i}$ and the background $\hat{x}_{i}$ must be negligible. Instead the general class of solutions (2.9), (2.10), (2.11) $A_{i}$ differs significantly from the fuzzy sphere $\hat{x}_{i}$ and cannot be used to define a consistent connection as in (2.12).

However we find fruitful exploring the neighborhood of $\lambda=\frac{1}{2}$, since the difference of the classical action computed on the class of solutions (2.11) and the classical action computed on the fuzzy sphere goes like $\left(\lambda-\frac{1}{2}\right)^{3}$, and can be negligible in the $N \rightarrow \infty$ limit if $\lambda$ scales as

$$
\lambda=\frac{1}{2}+\rho \lambda_{0}
$$

It is aim of this paper to show that this further constraint can be implemented to give non trivial connections $\hat{a}_{i}$, which then describe noncommutative solitons for the fuzzy sphere.

The first difficulty we meet is to find compatibility between the two constraints 


$$
\begin{aligned}
& \alpha^{2}+\beta^{2}+\gamma^{2}=4 \rho^{2}(1-\lambda)+4 \rho^{2} \lambda^{2} \\
& \lambda=\frac{1}{2}+\rho \lambda_{0}
\end{aligned}
$$

since the general solution for $A_{i}^{1}, A_{i}^{2}$ and $A_{i}^{3}$ is constructed uniquely in terms of $\alpha, \beta, \gamma$. To find compatibility, we need to parameterize $\alpha, \beta, \gamma$ as:

$$
\begin{aligned}
& \alpha=\rho+\alpha_{0} \rho^{2} \\
& \beta=\rho+\beta_{0} \rho^{2} \\
& \gamma=\rho+\gamma_{0} \rho^{2}
\end{aligned}
$$

from which it follows that

$$
\begin{aligned}
& \alpha_{0}+\beta_{0}+\gamma_{0}=0 \\
& \alpha_{0}^{2}+\beta_{0}^{2}+\gamma_{0}^{2}=4 \lambda_{0}^{2} .
\end{aligned}
$$

A general solution of this system is given by:

$$
\begin{aligned}
& \alpha_{0}=2 \sqrt{\frac{2}{3}} \lambda_{0} \sin \left(\frac{\pi}{3}-\theta\right) \\
& \beta_{0}=2 \sqrt{\frac{2}{3}} \lambda_{0} \sin \theta \\
& \gamma_{0}=-2 \sqrt{\frac{2}{3}} \lambda_{0} \sin \left(\frac{\pi}{3}+\theta\right) .
\end{aligned}
$$

This is not the whole story, since the general solution (2.11), to be well defined, must satisfy the following inequalities

$$
\alpha^{2} \geq 2 \rho^{2}(1-\lambda) \quad \beta^{2} \geq 2 \rho^{2}(1-\lambda) \quad \gamma^{2} \geq 2 \rho^{2}(1-\lambda) .
$$

By defining

$$
\sin \alpha_{0}=\frac{1}{2} \sqrt{\frac{3}{2}}
$$


these inequalities imply that

$$
\sin \left(\frac{\pi}{3}-\theta\right) \geq-\sin \alpha_{0} \quad \sin \theta \geq-\sin \alpha_{0} \quad \sin \left(\frac{\pi}{3}+\theta\right) \leq \sin \alpha_{0} .
$$

It is surprising to observe that there is a narrow window of values for $\theta$ which satisfies all these inequalities:

$$
\frac{4}{3} \pi-\alpha_{0}<\theta<\pi+\alpha_{0}
$$

In the following we will use a simple fixed value of $\theta$ to simplify the calculations :

$$
\theta=\frac{7}{6} \pi
$$

and the general solution to the constraints $(2.16)$ and $(2.20)$ is determined to be :

$$
\begin{aligned}
& \alpha=\rho\left(1-\sqrt{\frac{2}{3}} \lambda_{0} \rho\right) \\
& \beta=\rho\left(1-\sqrt{\frac{2}{3}} \lambda_{0} \rho\right) \\
& \gamma=\rho\left(1+2 \sqrt{\frac{2}{3}} \lambda_{0} \rho\right) .
\end{aligned}
$$

\section{Solitons for the fuzzy sphere}

In order to determine the solitons for the fuzzy sphere we have to recall how to recover gauge theory on the fuzzy sphere, by expanding the $A_{i}$ matrices around the classical solution (2.2) as:

$$
A_{i}=\hat{x}_{i}+\rho R \hat{a}_{i}
$$

The action $S(\lambda)(2.1)$ is invariant under the unitary transformation

$$
A_{i} \rightarrow U^{-1} A_{i} U
$$

which implements the gauge symmetry of the noncommutative gauge theories as a global symmetry of the matrix model. 
In fact, by developing $U$ in terms of an infinitesimal transformation

$$
U \sim 1+i \hat{\lambda}
$$

the fluctuations around the fixed background transforms as

$$
\hat{a}_{i} \rightarrow \hat{a}_{i}-\frac{i}{R}\left[\hat{L}_{i}, \hat{\lambda}\right]+i\left[\hat{\lambda}, \hat{a}_{i}\right]
$$

The corresponding field strength on the sphere is given by

$$
\begin{aligned}
\hat{F}_{i j} & =\frac{1}{\rho^{2} R^{2}}\left(\left[A_{i}, A_{j}\right]-i \rho \epsilon_{i j k} A_{k}\right) \\
& =\left[\frac{\hat{L}_{i}}{R}, \hat{a}_{j}\right]-\left[\frac{\hat{L}_{j}}{R}, \hat{a}_{i}\right]+\left[\hat{a}_{i}, \hat{a}_{j}\right]-\frac{i}{R} \epsilon_{i j k} \hat{a}_{k} .
\end{aligned}
$$

$\hat{F}_{i j}$ is gauge covariant even in the $U(1)$ case, as it is manifest from the viewpoint of the matrix model.

The model contains also a scalar field which belongs to the adjoint representation as the gauge field and that can be defined as

$$
\hat{\phi}=\frac{1}{2 \rho R}\left(A_{i} A_{i}-\hat{x}_{i} \hat{x}_{i}\right)=\frac{1}{2}\left(\hat{x}_{i} \hat{a}_{i}+\hat{a}_{i} \hat{x}_{i}+\rho R \hat{a}_{i} \hat{a}_{i}\right)
$$

At the noncommutative level, the scalar model is intrinsically connected with the gauge field and only in the classical limit the action can be interpreted as a sum of both contributions.

To define the action in terms of the fluctuations $\hat{a}_{i}$, we have to define a star product on the fuzzy sphere analogous to the Moyal star product for the plane.

Recall that a matrix on the fuzzy sphere can be developed in terms of the noncommutative analogue of the spherical harmonics $\hat{Y}_{l m}$ :

$$
\hat{Y}_{l m}=R^{-l} \sum_{a} f_{a_{1}, a_{2}, \ldots a_{l}}^{(l m)} \hat{x}_{a_{1}} \ldots \hat{x}_{a_{l}}
$$

while the classical spherical harmonics are defined with $\hat{x}_{i}$ substituted with the commutative coordinates $x_{i}$.

A general matrix 


$$
\hat{a}=\sum_{l=0}^{N} \sum_{m=-l}^{l} a_{l m} \hat{Y}_{l m} \quad a_{l m}^{*}=a_{l-m}
$$

corresponds therefore to an ordinary function on the commutative sphere as:

$$
a(\Omega)=\frac{1}{N+1} \sum_{l=0}^{N} \sum_{m=-l}^{l} \operatorname{Tr}\left(\hat{Y}_{l m}^{\dagger} \hat{a}\right) Y_{l m}(\Omega)
$$

and the ordinary product of matrices is mapped to the star product on the commutative sphere

$$
\begin{aligned}
& \hat{a} \hat{b} \rightarrow a * b \\
& a(\Omega) * b(\Omega)=\frac{1}{N+1} \sum_{l=0}^{N} \sum_{m=-l}^{l} \operatorname{Tr}\left(\hat{Y}_{l m}^{\dagger} \hat{a} \hat{b}\right) Y_{l m}(\Omega) .
\end{aligned}
$$

Derivative operators can be constructed using the adjoint action of $\hat{L}_{i}$ and tend to the classical Lie derivative $L_{i}$ in the $N \rightarrow \infty$ limit:

$$
A d\left(\hat{L}_{i}\right) \rightarrow L_{i}=\frac{1}{i} \epsilon_{i j k} x_{j} \partial_{k}
$$

$L_{i}$ can be expanded in terms of the Killing vectors of the sphere

$$
L_{i}=-i K_{i}^{a} \partial_{a}
$$

In terms of $K_{i}^{a}$ we can form the metric tensor $g_{a b}=K_{a}^{i} K_{b}^{i}$. The explicit form of these Killing vectors is

$$
\begin{aligned}
& K_{1}^{\theta}=-\sin \phi \quad K_{1}^{\phi}=-\operatorname{cotg} \theta \cos \phi \\
& K_{2}^{\theta}=\cos \phi \quad K_{2}^{\phi}=-\operatorname{cotg} \theta \sin \phi \\
& K_{3}^{\theta}=0 \quad K_{3}^{\phi}=1 .
\end{aligned}
$$

Trace over matrices can be mapped to the integration over functions:

$$
\frac{1}{N+1} \operatorname{Tr}(\hat{a}) \rightarrow \int \frac{d \Omega}{4 \pi} a(\Omega)
$$

Having introduced the star product, we can compute the action $S(\lambda)$ as the following field theory action 


$$
\begin{aligned}
& S(\lambda)=S_{0}+\lambda S_{1} \\
& S_{0}=-\frac{R^{2}}{4 g_{y m}^{2}} \operatorname{Tr} \int d \Omega\left(F_{i j} F_{i j}\right)-\frac{3 i}{2 g_{y m}^{2}} \epsilon_{i j k} \operatorname{Tr} \int d \Omega\left(\left(L_{i} a_{j}\right) a_{k}+\frac{R}{3}\left[a_{i}, a_{j}\right] a_{k}-\frac{i}{2} \epsilon_{i j l} a_{l} a_{k}\right)_{*} \\
& -\frac{\pi}{g_{y m}^{2}} \frac{N(N+2)}{2 R^{2}} \\
& S_{1}=\frac{i}{2 g_{y m}^{2}} \epsilon_{i j k} \operatorname{Tr} \int d \Omega\left(\left(L_{i} a_{j}\right) a_{k}+\frac{R}{3}\left[a_{i}, a_{j}\right] a_{k}-\frac{i}{2} \epsilon_{i j l} a_{l} a_{k}\right)_{*} \\
& +\frac{\pi}{3 g_{y m}^{2}} \frac{N(N+2)}{2 R^{2}}
\end{aligned}
$$

where the residual Trace is in general for the $U(n)$ case, defined as in [10]- 112.

Our solution $A_{i}$, defined by the equations (2.23) gives rise to a solution of the action $S(\lambda)$ and therefore is a soliton solution for the fuzzy sphere, since the fluctuations respect the dependence on $\rho$ of equation (2.12). However to help intuition we will look for the classical limit of this solution and verify that it is a nontrivial solution of the classical limit of the action $S(\lambda)$, i.e. a classical soliton for the sphere.

The classical limit is realized as

$$
R=\text { fixed } \quad g_{y m}^{2}=\frac{4 \pi g^{2}}{(N+1) \rho^{4} R^{2}}=\text { fixed } \quad N \rightarrow \infty .
$$

In the commutative limit, the star product becomes the commutative product. In this limit, the scalar field $\phi$ and the gauge field are separable from each other as in

$$
R a_{i}(\Omega)=K_{i}^{a} b_{a}(\Omega)+\frac{x_{i}}{R} \phi(\Omega)
$$

where $b_{a}$ is a gauge field on the sphere. The field strength $F_{i j}$ can be expanded in terms of the gauge field $b_{a}$ and the scalar field $\phi$ as follows

$$
F_{i j}(\Omega)=\frac{1}{R^{2}} K_{i}^{a} K_{j}^{b} F_{a b}+\frac{i}{R^{2}} \epsilon_{i j k} x_{k} \phi+\frac{1}{R^{2}} x_{j} K_{i}^{a} D_{a} \phi-\frac{1}{R^{2}} x_{i} K_{j}^{a} D_{a} \phi
$$

where $F_{a b}=-i\left(\partial_{a} b_{b}-\partial_{b} b_{a}\right)+\left[b_{a}, b_{b}\right]$ and $D_{a}=-i \partial_{a}+\left[b_{a}, \ldots\right]$, in general for the $U(n)$ case.

Let us identify the classical fluctuations $a_{i}$ in terms of the solution for $A_{i}$ (2.23), developed in power of $\rho$ : 


$$
\begin{aligned}
& A_{x}^{1}=\rho\left(1-\sqrt{\frac{2}{3}} \lambda_{0} \rho\right) \\
& A_{x}^{2}=-2 \lambda_{0}\left(1-\sqrt{\frac{2}{3}}\right) \rho^{2}\left(1-\sqrt{\frac{2}{3}} \lambda_{0} \rho\right)+O\left(\rho^{4}\right) \\
& A_{y}^{2}=\rho\left(1-\sqrt{\frac{2}{3}} \lambda_{0} \rho-2 \lambda_{0}^{2}\left(1-\sqrt{\frac{2}{3}}^{2} \rho^{2}\right)+O\left(\rho^{4}\right)\right. \\
& A_{x}^{3}=-2 \lambda_{0} \sqrt{\left(1-\sqrt{\frac{2}{3}}\right)\left(1+2 \sqrt{\frac{2}{3}}\right)} \rho^{2}\left(1+2 \sqrt{\frac{2}{3}} \lambda_{0} \rho\right)+O\left(\rho^{4}\right)
\end{aligned}
$$

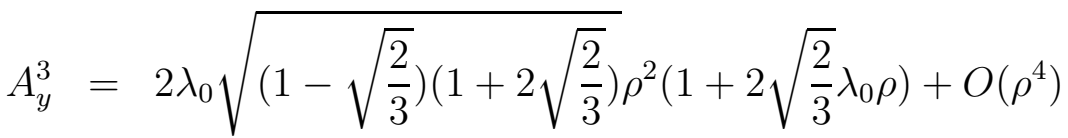

$$
\begin{aligned}
& A_{z}^{3}=\rho\left(1+2 \sqrt{\frac{2}{3}} \lambda_{0}-4 \lambda_{0}^{2}\left(1-\sqrt{\frac{2}{3}}\right)\left(1+2 \sqrt{\frac{2}{3}}\right) \rho^{2}\right)+O\left(\rho^{4}\right) .
\end{aligned}
$$

We have kept the first subleading contribution to the classical fluctuation $a_{i}$, since, as we will see in the next section, they can in principle give a finite contribution to the action $S(\lambda)$. The classical fluctuations $a_{i}$ are deducible from (3.19) as follows:

$$
\begin{aligned}
& R a^{1}=-\sqrt{\frac{2}{3}} \lambda_{0} x^{1} \\
& R a^{2}=-2 \lambda_{0}\left(1-\sqrt{\frac{2}{3}}\right) x_{1}-\sqrt{\frac{2}{3}} \lambda_{0} x_{2} \\
& R a_{3}=-2 \lambda_{0} \sqrt{\left(1-\sqrt{\frac{2}{3}}\right)\left(1+2 \sqrt{\frac{2}{3}}\right)\left(x_{1}-x_{2}\right)+2 \sqrt{\frac{2}{3}} \lambda_{0} x_{3} .}
\end{aligned}
$$

The classical scalar field, obtained from this particular fluctuation $a_{i}$, is :

$$
R \phi(\Omega)=x_{i} \cdot R a_{i}
$$

therefore

$$
\begin{aligned}
R \phi(\Omega) & =\sqrt{\frac{2}{3}} \lambda_{0}\left(x^{1}\right)^{2}-2 \lambda_{0}\left(1-\sqrt{\frac{2}{3}}\right) x^{1} x^{2}-\sqrt{\frac{2}{3}}\left(x^{2}\right)^{2} \\
& -2 \lambda_{0} \sqrt{\left(1-\sqrt{\frac{2}{3}}\right)\left(1+2 \sqrt{\frac{2}{3}}\right)\left(x^{1}-x^{2}\right) x^{3}} \\
& +2 \sqrt{\frac{2}{3}} \lambda_{0}\left(x^{3}\right)^{2}
\end{aligned}
$$


or, in usual spherical coordinates,

$$
\begin{aligned}
\phi(\Omega) & =R\left[\lambda_{0} \sqrt{\frac{2}{3}}\left(2 \cos ^{2} \theta-\sin ^{2} \theta\right)-2 \lambda_{0}\left(1-\sqrt{\frac{2}{3}}\right) \sin ^{2} \theta \sin \phi \cos \phi\right. \\
& \left.\left.+2 \lambda_{0} \sqrt{\left(1-\sqrt{\frac{2}{3}}\right)\left(1+2 \sqrt{\frac{2}{3}}\right.}\right) \sin \theta \cos \theta(\sin \phi-\cos \phi)\right] .
\end{aligned}
$$

From the explicit form of the Killing vector $K_{i}^{a}$, we can deduce the two components of the $U(1)$ gauge field as:

$$
\begin{aligned}
b_{\theta} & =-R a^{1} \sin \phi+R a^{2} \cos \phi= \\
& =-2 R \lambda_{0}\left(1-\sqrt{\frac{2}{3}}\right) \sin \theta \cos ^{2} \phi
\end{aligned}
$$

and

$$
\begin{aligned}
b_{\phi} & =R\left[2 \lambda_{0} \sqrt{\left(1-\sqrt{\frac{2}{3}}\right)\left(1+2 \sqrt{\frac{2}{3}}\right.}\right) \sin ^{3} \theta(\sin \phi-\cos \phi) \\
& \left.+3 \lambda_{0} \sqrt{\frac{2}{3}} \cos \theta \sin ^{2} \theta+2 \lambda_{0}\left(1-\sqrt{\frac{2}{3}}\right) \sin ^{2} \theta \cos \theta \sin \phi \cos \phi\right] .
\end{aligned}
$$

Having made contact with the soliton solution, we now verify that it is a non trivial solution of the classical equations of motion on the sphere.

The classical limit of the action $S(\lambda)$ is determined to be:

$$
\begin{aligned}
S(\lambda) & =S_{0}+\lambda S_{1} \\
S_{0} & =-\frac{1}{4 g_{y m}^{2} R^{2}} \operatorname{Tr} \int d \Omega\left(K_{i}^{a} K_{j}^{b} K_{i}^{c} K_{j}^{d} F_{a b} F_{c d}+2 i K_{i}^{a} K_{j}^{b} F_{a b} \epsilon_{i j k} \frac{x_{k}}{R} \phi\right. \\
& \left.+2 K_{i}^{a} K_{i}^{b}\left(D_{a} \phi\right)\left(D_{b} \phi\right)-2 \phi^{2}\right) \\
& -\frac{3}{2 g_{y m}^{2} R^{2}} \operatorname{Tr} \int d \Omega\left(i \epsilon_{i j k} K_{i}^{a} K_{j}^{b} F_{a b} \frac{x_{k}}{R} \phi-\phi^{2}\right) \\
& =-\frac{1}{4 g_{y m}^{2} R^{2}} \operatorname{Tr} \int d \Omega\left(F_{a b} F^{a b}+8 i \frac{\epsilon^{a b}}{\sqrt{g}} F_{a b} \phi+2\left(D_{a} \phi\right)\left(D^{a} \phi\right)-8 \phi^{2}\right) \\
S_{1} & =\frac{1}{g_{y m}^{2} R^{2}} \operatorname{Tr} \int d \Omega\left(i \epsilon_{i j k} K_{i}^{a} K_{j}^{b} F_{a b} \frac{x_{k}}{R} \phi-\phi^{2}\right)= \\
& =\frac{1}{g_{y m}^{2} R^{2}} \operatorname{Tr} \int d \Omega\left(\frac{i \epsilon^{a b}}{\sqrt{g}} F_{a b} \phi-\phi^{2}\right)
\end{aligned}
$$


where $\epsilon^{a b}$ is defined as $\epsilon^{\theta \phi}=1$.

Since we are interested in the $U(1)$ case, we finally find

$$
S(\lambda)=S_{0}+\lambda S_{1}=-\frac{1}{4 g_{y m}^{2} R^{2}} \int d \Omega\left[F_{a b} F^{a b}-2 \partial_{a} \phi \partial_{a} \phi+(8-4 \lambda)\left(\frac{i \epsilon^{a b}}{\sqrt{g}} F_{a b} \phi-\phi^{2}\right)\right] .
$$

As in the Bogomolnyi trick, we can isolate an equation of motion directly at the level of action as a quadratic term

$$
\begin{aligned}
S(\lambda) & =-\frac{1}{4 g_{y m}^{2} R^{2}} \int d \Omega\left[\left(F_{a b}+(4-2 \lambda) i \epsilon_{a b} \phi \sqrt{g}\right)\left(F^{a b}+(4-2 \lambda) i \epsilon^{a b} \frac{\phi}{\sqrt{g}}\right)\right. \\
& \left.-2 \partial_{a} \phi \partial_{a} \phi+\left[2(4-2 \lambda)^{2}-4(2-\lambda)\right] \phi^{2}\right]
\end{aligned}
$$

from which the resulting equations of motion are

$$
\begin{aligned}
& F_{a b}+(4-2 \lambda) i \epsilon_{a b} \phi \sqrt{g}=0 \\
& \partial^{a} \partial_{a} \phi=L_{i} L_{i} \phi=\left[(4-2 \lambda)^{2}-2(2-\lambda)\right] \phi .
\end{aligned}
$$

We can recognize in the second equation the eigenvalue problem for the $L_{i} L_{i}$ operator which admits nontrivial solutions if and only if

$$
L_{i} L_{i} \phi=l(l+1) \phi
$$

the coefficient in front of $\phi$ in the second member is equal to $l(l+1)$. By imposing that

$$
l(l+1)=(4-2 \lambda)^{2}-2(2-\lambda)
$$

we find that the only classical models which admit nontrivial solutions are for

$$
\lambda=\frac{3-l}{2} \quad \text { or } \quad \lambda=\frac{l+4}{2}
$$

The case $l=0$, i.e. a constant scalar field $\phi$ can be reached with the method outlined in the Appendix. 
From the formula (2.23), the scalar field $\phi(\Omega)$ is a combination of $Y_{2}^{m}$ spherical harmonics, and we can verify that at $l=2 \lambda=\frac{1}{2}$.

Therefore, one equation of motion is surely satisfied. To check also the other equation we need to compute $F_{\theta \phi}$

$$
\begin{aligned}
F_{\theta \phi} & =-i\left(\partial_{\theta} b_{\phi}-\partial_{\phi} b_{\theta}\right)= \\
& -3 i \sin \theta R\left[2 \lambda_{0} \sqrt{\left(1-\sqrt{\frac{2}{3}}\right)\left(1+2 \sqrt{\frac{2}{3}}\right) \sin \theta \cos \theta(\sin \phi-\cos \phi)}\right. \\
& +\lambda_{0} \sqrt{\frac{2}{3}} \sin \theta\left(2 \cos ^{2} \theta-\sin ^{2} \theta-2 \lambda_{0}\left(1-\sqrt{\frac{2}{3}}\right) \sin ^{2} \theta \cos \phi \sin \phi\right]= \\
& =-3 i \sin \theta \phi(\Omega) \epsilon_{\theta \phi}
\end{aligned}
$$

which satisfies the other equations of motion for $\lambda=\frac{1}{2}$.

Therefore our solution is a noncommutative soliton for the fuzzy sphere which, in the $N \rightarrow \infty$ limit, corresponds to a classical soliton for the sphere with the classical action $S\left(\frac{1}{2}\right)$.

To find noncommutative solitons with higher spherical harmonics $l>2$ one should explore the neighborhood of the models

$$
\lambda=\frac{3-l}{2}
$$

by starting from classical solutions of matrix model obtained with more general ansatze:

$$
A_{i}=A_{i}^{j_{1}, j_{2}, . ., j_{l-1}} L_{j_{1}} \ldots L_{j_{l-1}}
$$

\section{Computation of the action}

To finish our verification, let us compare the action $S(\lambda)$ evaluated at the solution $A_{i}$ with the action evaluated on the classical soliton on the sphere.

Recall that the computation of the action $S(\lambda)$ on the general class of solutions (2.9), (2.10), (2.11) is made of three pieces:

$$
\operatorname{Tr}\left[A^{i}, A^{j}\right]\left[A^{i}, A^{j}\right]=-\frac{8}{3} \rho^{4}(1-\lambda)\left(4 \lambda^{2}-\lambda+1\right) \operatorname{Tr}\left(\hat{L}_{m} \hat{L}_{m}\right)
$$




$$
\begin{aligned}
& -\frac{2}{3} i \lambda \rho \operatorname{Tr} \epsilon_{i j k} A^{i} A^{j} A^{k}=\frac{8}{3} \lambda^{2}(1-\lambda) \rho^{4} \operatorname{Tr}\left(\hat{L}_{m} \hat{L}_{m}\right) \\
& \rho^{2}(1-\lambda) \operatorname{Tr} A^{i} A^{i}=\frac{4}{3} \rho^{4}(1-\lambda)\left(\lambda^{2}-\lambda+1\right) \operatorname{Tr}\left(\hat{L}_{m} \hat{L}_{m}\right) .
\end{aligned}
$$

The total evaluation of the action $S(\lambda)$ is therefore given by:

$$
\left.S(\lambda)\right|_{\text {tot }}=S_{0}+\lambda S_{1}=-\frac{1}{3 g^{2}} \rho^{4}(1-\lambda)\left(2-2 \lambda+4 \lambda^{2}\right) \operatorname{Tr}\left(\hat{L}_{m} \hat{L}_{m}\right) .
$$

This action contains a part which is divergent in the $N \rightarrow \infty$ limit, and the remaining part which is finite. Subtracting the divergent part we find

$$
\left.S(\lambda)\right|_{\text {finite }}=\left.S(\lambda)\right|_{\text {tot }}-\left.S(\lambda)\right|_{\text {div }}=\frac{4 \rho^{4}}{3 g^{2}}\left(\lambda-\frac{1}{2}\right)^{3} \operatorname{Tr}\left(\hat{L}_{i} \hat{L}_{i}\right) .
$$

By introducing the models $\lambda=\frac{1}{2}+\rho \lambda_{0}$, considered in this paper, we find:

$$
\left.S(\lambda)\right|_{\text {finite }}=\frac{4}{3 g^{2}} \rho^{7} \lambda_{0}^{3} \operatorname{Tr}\left(\hat{L}_{i} \hat{L}_{i}\right)=\frac{16 \pi \lambda_{0}^{3}}{3 R^{2} g_{y m}^{2}} \rho \rightarrow 0 \quad \text { for } \quad N \rightarrow \infty
$$

i.e. the finite value of the action $S(\lambda)$ vanishes as $\rho$ in the $N \rightarrow \infty$ limit.

Before comparing this null result with the value of the classical action at the classical soliton, we shall compute the possible finite value of the subleading term into the fluctuations, i.e. terms of order $\rho$ with respect to the classical solitons, to the action $S(\lambda)$.

For example, let us take the term

$$
\operatorname{Tr}\left[A^{i}, A^{j}\right]\left[A^{i}, A^{j}\right]=-\frac{8}{3} \rho^{4}(1-\lambda)\left(4 \lambda^{2}-\lambda+1\right) \operatorname{Tr}\left(\hat{L}_{i} \hat{L}_{)} .\right.
$$

By substituting the value $\lambda=\frac{1}{2}+\rho \lambda_{0}$, the total contribution, divergent plus finite, is given by

$$
\left.\operatorname{Tr}\left[A^{i}, A^{j}\right]\left[A^{i}, A^{j}\right]\right|_{\text {total }}=-\frac{8}{3} \rho^{4}\left(\frac{3}{4}-\rho^{2} \lambda_{0}^{2}\right) \operatorname{Tr}\left(\hat{L}_{i} \hat{L}_{i}\right) .
$$

However the contribution of the leading fluctuations $a_{i}$, which define the classical solitons, is different

$$
\left.\operatorname{Tr}\left[A^{i}, A^{j}\right]\left[A^{i}, A^{j}\right]\right|_{\text {soliton }}=-\frac{8}{3} \rho^{4}\left(\frac{3}{4}+\rho^{2} \lambda_{0}^{2}\right) \operatorname{Tr}\left(\hat{L}_{i} \hat{L}_{i}\right) .
$$


The difference is given by the subleading fluctuations $\tilde{a}_{i} \sim O(\rho)$, which vanish in the classical limit,

$$
\left.\operatorname{Tr}\left[A^{i}, A^{j}\right]\left[A^{i}, A^{j}\right]\right|_{s u b}=\frac{16}{3} \rho^{6} \lambda_{0}^{2} \operatorname{Tr}\left(\hat{L}_{i} \hat{L}_{i}\right)
$$

Analogously the other two pieces of action, evaluated on the total solution (2.23), on the leading fluctuations (3.20) and on the subleading ones lead to

$$
\begin{aligned}
& \left.\epsilon^{i j k} \operatorname{Tr} A^{i}\left[A^{j}, A^{k}\right]\right|_{\text {total }}=2 i \rho^{3}\left(1-4 \lambda_{0}^{2} \rho^{2}\right) \operatorname{Tr}\left(\hat{L}_{i} \hat{L}_{i}\right) \\
& \left.\epsilon^{i j k} \operatorname{Tr} A^{i}\left[A^{j}, A^{k}\right]\right|_{\text {soliton }}=2 i \rho^{3}\left(1-2 \lambda_{0}^{2} \rho^{2}\right) \operatorname{Tr}\left(\hat{L}_{i} \hat{L}_{i}\right) \\
& \left.\epsilon^{i j k} \operatorname{Tr} A^{i}\left[A^{j}, A^{k}\right]\right|_{\text {sub }}=-4 i \lambda_{0}^{2} \rho^{5} \operatorname{Tr}\left(\hat{L}_{i} \hat{L}_{i}\right) \\
& \left.\operatorname{Tr} A^{i} A^{i}\right|_{\text {total }}=\frac{4}{3} \rho^{2}\left[\frac{3}{4}+\lambda_{0}^{2} \rho^{2}\right] \operatorname{Tr}\left(\hat{L}_{i} \hat{L}_{i}\right) \\
& \left.\operatorname{Tr} A^{i} A^{i}\right|_{\text {soliton }}=\frac{4}{3} \rho^{2}\left[\frac{3}{4}+2 \lambda_{0}^{2} \rho^{2}\right] \operatorname{Tr}\left(\hat{L}_{i} \hat{L}_{i}\right) \\
& \left.\operatorname{Tr} A^{i} A^{i}\right|_{\text {sub }}=-\frac{4}{3} \rho^{4} \lambda_{0}^{2} \operatorname{Tr}\left(\hat{L}_{i} \hat{L}_{i}\right) .
\end{aligned}
$$

Fortunately, these finite contributions of the subleading fluctuations cancel out from the action $S(\lambda)$. In fact for $S_{0}$ and $S_{1}$ we find

$$
\begin{aligned}
& S_{0} \propto\left(\frac{1}{4} \frac{16}{3} \rho^{6}-\frac{4}{3} \rho^{6}\right) \lambda_{0}^{2} \operatorname{Tr}\left(\hat{L}_{i} \hat{L}_{i}\right)=0 \\
& S_{1} \propto\left(\frac{4}{3} \rho^{6}-\frac{4}{3} \rho^{6}\right) \lambda_{0}^{2} \operatorname{Tr}\left(\hat{L}_{i} \hat{L}_{i}\right)=0 .
\end{aligned}
$$

We conclude that, since the total action $S(\lambda)$, evaluated on the complete solution $A_{i}$, is vanishing in the $N \rightarrow \infty$ limit, and since the subleading terms give no extra contribution, the value of the classical action on the classical solitons must be zero.

In fact for the classical action (3.28) the equation of motion

$$
F_{a b}+3 i \epsilon_{a b} \sqrt{g} \phi=0
$$

cancels the quadratic part of the action, while the other equation

$$
\partial^{a} \partial_{a} \phi=6 \phi
$$

cancels also the other part, since the action is homogeneously quadratic in $\phi$ : 


$$
\left.S\left(\frac{1}{2}\right)\right|_{\text {soliton }}=0 .
$$

However the solution is clearly non trivial, since $F_{a b}$ cannot be made equivalent to zero with a gauge transformation. Topological arguments as Chern - classes can eventually give rise to a quantization of the parameter $\lambda_{0}$, which we have left undetermined.

\section{Conclusion}

In this paper we have searched for non trivial solutions to the noncommutative gauge theory over the fuzzy sphere. Between all the possible solutions of the matrix model, those which have the right to be taken into account are those which corresponds to the classical solitons on a sphere in the $N \rightarrow \infty$ limit.

The fluctuations, defined as the difference between the generic solutions of the matrix model and the background ( the fuzzy sphere), must vanish in the $N \rightarrow \infty$ limit as $\frac{1}{N}$, a constraint which implies a double scaling limit in the two coupling constants of the model, $g^{2}$ and $\lambda$. Therefore to find classical solitons we have to scale $\lambda$ as $\frac{1}{2}+\frac{\lambda_{0}}{N}$.

By analyzing the classical solitons we find confirmations of the scheme proposed, since the scalar field corresponding to our classical solution is a combination of the spherical harmonics $l=2$. This should be expected since the classical solution of the matrix model we started from were found by the ansatz

$$
A^{i}=A_{j}^{i} L^{j}
$$

There is in fact a correspondence between the model $\lambda=\frac{1}{2}$ and the spherical harmonics $l=2$. By analogy, one could search classical solitons with higher spherical harmonics $l>2$, and therefore define new classes of noncommutative solitons over the fuzzy sphere. One should firstly start from more general ansatze:

$$
A^{i}=A_{j_{1}, j_{2}, \ldots, j_{l-1}}^{i} L^{j_{1}, j_{2}, \ldots, j_{l-1}}
$$

and determine the corresponding classical solutions of the matrix model. Then it should happen that these solutions are a smooth deformation of a fuzzy sphere around the models $\lambda=\frac{l-3}{2}$; finally to find the classical solitons with higher spherical harmonics one should scale $\lambda$ as $\frac{l-3}{2}+\frac{\lambda_{0}}{N}$. 
The solitons we have found are different from the monopoles, which are the standard solitons of gauge theory on the sphere. However the classical theory defined from the matrix model is more complex, being the sum of the gauge field and scalar field actions. In fact the value of the action on these classical solitons is zero and it is not proportional to the soliton number as it happens with the instantons on the plane [26]-27]-228]- 29]-[30]-31]- 332]. However we find another signals, typical of solitons, i.e. that the action contains an equation of motion quadratically, as it happens with vortices.

Finally, by keeping $\lambda=\frac{1}{2}+\frac{\lambda_{0}}{N}$ with $N$ finite, our new class of solutions determines a series of noncommutative solitons over the fuzzy sphere which converges to a classical soliton in the $N \rightarrow \infty$ limit.

We believe that the method here outlined is a practical procedure to define noncommutative solitons as a fuzzy sphere, and that the matrix model approach greatly simplify the task.

It is simpler to define the noncommutative soliton as a difference between two solutions of the matrix model, rather than trying to solve the equations of motion of the noncommutative gauge theory directly. It is an open question to find the quantum contribution of this solitons to the partition function, and again this question greatly simplifies if posed in the matrix model approach.

\section{A Appendix}

To obtain a constant scalar field as classical solution one starts with the ansatz

$$
A^{i}=f(\rho) L^{i}=\frac{f(\rho)}{\rho} \hat{x}^{i}
$$

i.e. a deformation with a scale factor of the standard fuzzy sphere solution. By imposing the equations of motion (2.5), one obtains the following quadratic form in $f(\rho)$ :

$$
f^{2}(\rho)-\lambda \rho f(\rho)-\rho^{2}(1-\lambda)=0
$$

whose solutions are

$$
\begin{aligned}
& f_{1}(\rho)=\rho \quad \text { standard fuzzy sphere } \\
& f_{2}(\rho)=(\lambda-1) \rho \quad \text { rescaled fuzzy sphere. }
\end{aligned}
$$


The case $\lambda=-1$ of the rescaled fuzzy sphere coincides with the case $\lambda=-1$ of the class of solutions considered in the paper.

Let us notice that for $\lambda=2$ the two solutions coincide and the value of the classical action $S(\lambda)$ around $\lambda=2$ is

$$
\begin{aligned}
& \left.S(\lambda)\right|_{2}=\left.\frac{\rho^{4}}{2 g^{2}}(\lambda-1)^{3}\left(1-\frac{\lambda}{3}\right) \operatorname{Tr}\left(\hat{L}_{i} \hat{L}_{i}\right) \quad S(\lambda)\right|_{\text {fuzzy sphere }}=-\frac{\rho^{4}}{2 g^{2}}\left(\frac{1}{2}-\frac{\lambda}{3}\right) \operatorname{Tr}\left(\hat{L}_{i} \hat{L}_{i}\right) \\
& \left.S(\lambda)\right|_{2}-\left.S(\lambda)\right|_{\text {fuzzy sphere }}=-\frac{\rho^{4}}{6 g^{2}} \lambda(\lambda-2)^{3} \operatorname{Tr}\left(\hat{L}_{i} \hat{L}_{i}\right) .
\end{aligned}
$$

By expanding $\lambda=2+\rho \lambda_{0}$ one then builds, in the same way as considered in the paper, noncommutative solitons which tend to a constant scalar field $\phi=c$ and null field strength $F_{a b}=0$, in the classical limit.

\section{References}

[1] E. Witten, " Noncommutative geometry and string field theory ", Nucl. Phys. B268 (1986) 253.

[2] N. Seiberg and E. Witten, " String Theory and Noncommutative geometry ", JHEP 9909 (1999) 032, hep-th/9908142.

[3] A. Connes, M. R. Douglas and A. Schwarz, " Noncommutative geometry and Matrix theory: compactification on Tori ", JHEP 9802 (1998) 003, hep-th/9711162.

[4] J. Ambjorn, Y.M. Makeenko, J. Nishimura and R. J. Szabo, " Finite N Matrix models of noncommutative gauge theory", JHEP 9911 (1999) 029, hep-th/9911041.

[5] J. Ambjorn, Y.M. Makeenko, J. Nishimura and R. J. Szabo, " Non perturbative Dynamics of noncommutative gauge theory ", Phys. Lett. B480 (2000) 399.

[6] J. Ambjorn, Y.M. Makeenko, J. Nishimura and R. J. Szabo, " Lattice gauge fields and discrete noncommutative Yang-Mills Theory ", JHEP 0005 (2000) 023, hepth/0004147.

[7] T. Eguchi and K. Kawai, " Reduction of dynamical degrees of freedom in the large N gauge theory", Phys. Rev. Lett. 48 (1982) 1063.

[8] A. Gonzales-Arroyo and M. Okawa, " The twisted Eguchi-Kawai model: a reduced model for large N lattice gauge theory", Phys. Rev. D27 (1983) 2397. 
[9] N. Ishibashi, H. Kawai, Y. Kitazawa and A. Tsuchiya, " A large N reduced model as superstring ", Nucl. Phys. B498 (1997) 467, hep-th/9612115.

[10] S. Iso, Y. Kimura, K. Tanaka and K. Wakatsuki, " Noncommutative gauge theory on fuzzy sphere from Matrix model ", Nucl. Phys. B604 (2001) 121, hep-th/0101102.

[11] R.C. Myers, "Dielectric branes", JHEP 9912 (1999) 022, hep-th/9910053.

[12] Y. Kimura, " Noncommutative gauge theories on Fuzzy sphere and Fuzzy torus from Matrix model ", Prog. Theor. Phys. 106 (2001) 445, hep-th/0103192.

[13] D. Berenstein, J. M. Maldacena, H. Nastase, " Strings in flat space and PP waves from N=4 Superyang-mills" , JHEP 0204 (2002) 013, hep-th/0202021.

[14] P. Valtancoli, "Stability of the fuzzy sphere solution from matrix model, hepth/0206075.

[15] J. Madore, " The Fuzzy sphere ", Class. Quantum Grav. 9 (1992) 69.

[16] U. Carow-Watamura and S. Watamura, " Noncommutative geometry and gauge theory on Fuzzy sphere ", Comm. Math. Phys. 212 (2000) 395, hep-th/9801195.

[17] U. Carow-Watamura and S. Watamura, " Differential calculus on fuzzy sphere and scalar field ", Int. J. Mod. Phys. A13 (1998) 3235, q-alg/9710034.

[18] U. Carow-Watamura and S. Watamura, " Chirality and Dirac operator on Noncommutative sphere ". Comm. Math. Phys. 183 (1997) 365, hep-th/9605003.

[19] C. Klimcik, " Gauge theories on the noncommutative sphere ", Comm. Math. Phys. 199 (1998) 257, hep-th/9710153.

[20] H. Grosse and P. Presnajder, " The Dirac operators on the fuzzy sphere ", Lett. math. Phys. 33 (1995) 171.

[21] S. Baez, A.P. Balachandran, S. Vaidya, " Monopoles and Solitons in fuzzy physics ", Comm. Math. Phys. 208 (2000) 787,hep-th/9811169.

[22] A.P. Balachandran and S. Vaidya, " Instantons and chiral anomaly in fuzzy physics ", hep-th/9910129.

[23] A. P. Balachandran, X.Martin and D. O' Connor, " Fuzzy actions and their continuum limits ", hep-th/0007030. 
[24] P. Valtancoli, " Projectors for the fuzzy sphere " Mod.Phys.Lett. A16 (2001) 639, hep-th/0101189.

[25] S. Vaidya, " Scalar multi-solitons on the fuzzy sphere ", JHEP 0201 (2002) 011, hepth/0109102.

[26] N. Nekrasov, A. Schwarz, " Instantons on noncommutative $R^{4}$ and $(2,0)$ superconformal six dimensional theory", Comm. Math. Phys. 128 (1998) 689.

[27] D. J. Gross, N. A. Nekrasov, " Solitons in noncommutative gauge theory ", JHEP 0103 (2001) 044,hep-th/0010090.

[28] D. J. Gross, N. A. Nekrasov, " Monopoles and strings in noncommutative gauge theory ", JHEP 0007 (2000) 034, hep-th/0005204.

[29] K. Furuuchi, " Instantons on noncommutative $R^{4}$ and projection operators ", Prog. Theor. Phys. 103 (2000) 1043, hep-th/9912047.

[30] L. D. Paniak, R. J. Szabo, " Instanton expansion of noncommutative gauge theory in two dimensions ", hep-th/0203166.

[31] J. A. Harvey, P. Kraus, F. Larsen, " Exact noncommutative solitons ", JHEP 0012 (2000) 024, hep-th/0010060.

[32] P. Krauss and M. Shigemori, " Noncommutative Instantons and the Seiberg-Witten Map ", JHEP 0206 (2002) 034, hep-th/0110035. 